\title{
HYBRID SURGERY - THE WAY TOWARDS NOTES THE CHALLENGE FOR COMPUTER SCIENCE
}

\author{
H. Feussner. S. Can, A. Fiolka, A. Schneider \\ Workgroup for Minimally Invasive Interdisciplinary Therapeutic Interventions MITI, \\ Department of Surgery, Klinikum rechts der Isar der TU München, Ismaninger Str. 22, 81675 München
}

\begin{abstract}
It is acknowledged by the medical community that further development of minimally invasive surgery is mainly driven by progress in medical engineering. To overcome many of the current barriers, computer science has to play a major role. In particular, improvement of spatial orientation, the development of intelligent mechatronic support systems, intelligent workflow organization, and simulation are among the essential issues.
\end{abstract}

Index Terms - NOTES, situation awareness, simulation workflow, spatial orientation

\section{INTRODUCTION}

The evolution of modern minimally invasive surgery is increasingly depending upon innovative developments in biomedical engineering. Computer science (CS) will also play a crucial role.

This holds particularly true for the new surgical approach of Natural Orifice Transluminal Endoscopic Surgery (NOTES) or scarless surgery. This new technique implies surgery performed endoscopically by initially passing the endoscope through natural orifices like the mouth or the rectum and then transluminally into the abdomen, which would not otherwise be accessible using the endoscope. This is achieved by the intentional creation of perforations through the wall of the gastroinstestinal tract. The theoretical advantages of NOTES over the open or the laparoscopic approach include less invasiveness without postoperative pain, illumination of any abdominal lesion, and reduction in postoperative abdominal wall wound infection, hernia formation and adhesions.

However, this new approach is still far from being mature for routine clinical use and most surgical interventions are still confined to the animal laboratory. A considerable amount of work still has to be done for wider acceptance of this approach.

Surgeons and gastroenterologists soon recognized the essential importance of technological support and defined the most urgent needs. These were summarized in a report ("white paper") [1]. Several key issues from the white paper touch upon current barriers for NOTES that directly or indirectly concern CS including:

- Spatial orientation

- Mechatronic support systems

- Situation awareness

- Simulation

\section{SPATIAL ORIENTATION}

Endoluminal surgeons and intracavitary surgeons live in different worlds with a completely different perception of the anatomy. Laparoscopists are accustomed to the "anatomical horizon", whereas endoscopists are familiar with image-guided manipulation. Accordingly, two different images should be presented: The native view of the operative site is provided for the endoscopist, but compensation of the horizon (electronic image stabilization/ inversion) is most favourable for the laparoscopist.

In hybrid surgery, diaphanoscopy is currently the only means to detect the position of the endoscope. Navigation of the tips of both instruments facilitates the cooperation between "in" and "out". A reliable spatial orientation would be extremely helpful in identifying the optimal entry point prior to entering the peritoneal cavity with the trocar, if a laparoscopy is performed, or via the stomach during NOTES [2]. Dangerous anatomical structures such as larger vessels could be indicated and "No Go" areas could be defined. Admittedly, navigation and referencing in a highly dynamic area like the abdomen where organs can deform and move with respiration is far more demanding than in rigid anatomy such as the skull and bones. Nevertheless, these problems have to be solved to improve the surgical state of the art and some promising approaches have already been presented.

\section{MECHATRONIC SUPPORT SYSTEMS}

The trend towards single port surgery and, in particular, NOTES, stimulates the development of new flexible manipulation systems. Currently available laparoscopic 
instruments or flexible endoscopes are inadequate to perform important manoeuvres when manipulating tissues and to set up traction/ counter traction for exposure and division of anatomical structures. While building the hardware is one of the problems, some systems, like the Octopus [3] or our own "Highly versatile single port system" are already in experimental evaluation (Fig. 1).

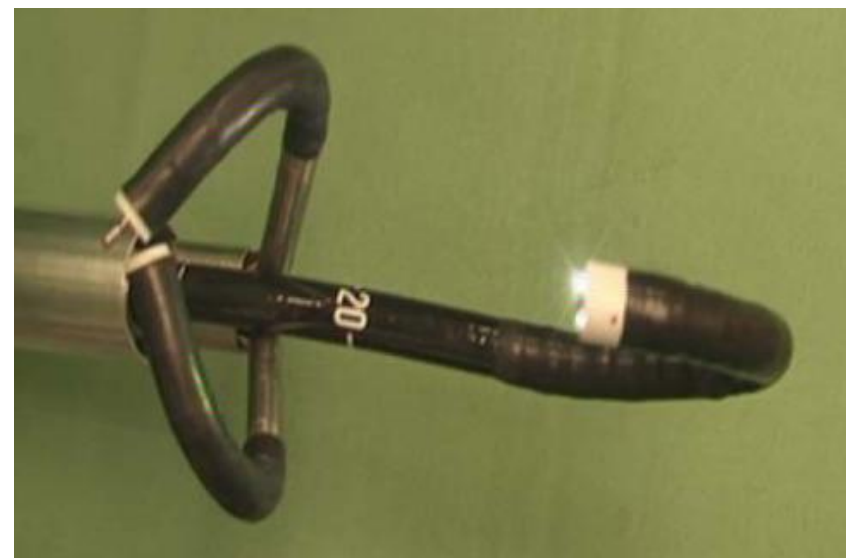

Fig. 1: End-effectors and flexible telescope of the "highly versatile single port system" (HVSPS) in a retroflexed working position. The significantly augmented workspace allows for completely new surgical approaches but multiplies the cognitive workload of the user (MITI).

Serious problems, however, arise as soon as the surgeon or the surgical team plan to work with mechatronic support systems under clinical conditions. The control of multiple devices and functions is excessively demanding. It is inconceivable that humans can master this task without intelligent support of something like an autopilot in aircraft industry. Two critical interfaces have to be considered: 1) the interface between anatomy and the machine, and 2) the interface between the machine and the human operator.

It is the task of computer science to provide safety and efficiency for manipulating the tissue with mechanic endeffectors by creating automated actions, identifying and avoiding potentially dangerous situations, etc. CS can improve the use of this type of complex technical system by supporting decision making and providing help to the user in performing the required manipulations.

\section{SITUATION AWARNESS}

The increasingly complicated interaction of the surgical team and the technical environment with multiple technical systems in hybrid operations and NOTES make it necessary to design the intelligent surgery room.

Based upon real time patient monitoring and online information about the state of surgical systems and of the respective surgical manipulation, continuous online workflow analysis should be achieved [4]. Thus, the future course of the surgery should be predictable, adverse events should be detected, and adaptive responses to breakdown in normal processes should be prepared [5]. The intraoperative collection should be performed in a stealth mode as described by Sutherland: "By stealth mode, we mean automated collection of data that is normally observed; yet irregularly captured because of lack of time and tedious manual data entry procedures. It is essential data for managing operations in a high stress environment, where erroneous perceptions generate suboptimal organizational response."

We have just begun to learn that NOTES per se may induce significant physiological changes which do not occur in laparoscopy and may endanger the patient even if the course of the surgery is "normal" (Fig. 2).

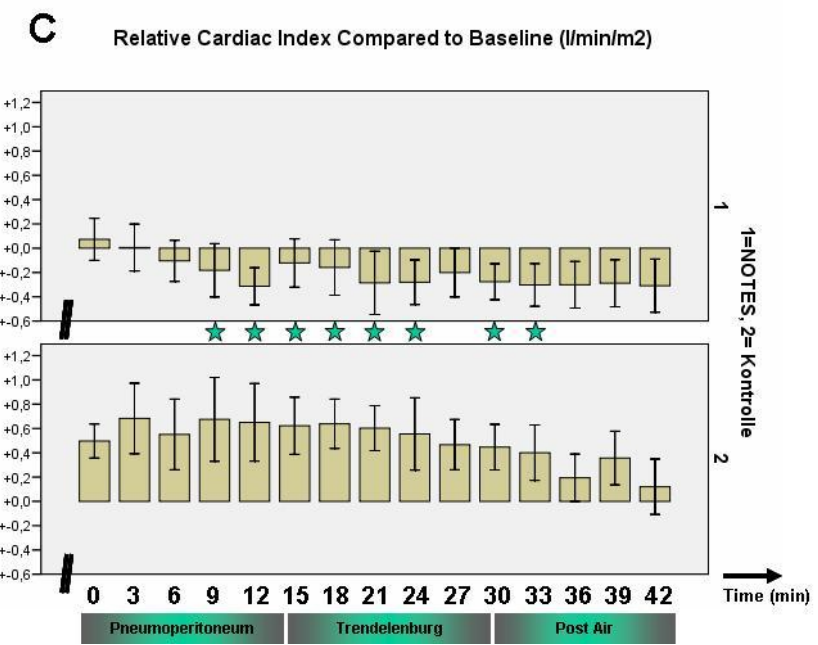

Fig. 2: Relative Cardiac Index during NOTES surgery (above) and laparoscopic surgery (below). During NOTES a significant reduction is demonstrated [6].

Reliable workflow analysis and interpretation, and, even a predictive capability in surgery are difficult, but essential. The first promising results have been reported [7] and it is no longer a matter of "if" but of "when" integrated intelligent interventional work places will be realized.

\section{SIMULATION}

It is a peculiarity of hybrid operations and NOTES that both the cognitive abilities and manual skills of an endoscopist and of an experienced surgeon are required. Accordingly, teamwork and communication between the two parties is critical. At present, practical training is only possible in animals. Training manikins like the ELITE [8] are in evaluation, but in the long term, training systems based on virtual reality should be provided. As in minor 
access surgery or endoscopy [9], these trainers are essential to improve and shorten the way from bench to bedside.

\section{HYBRID SURGERY}

As shown above multiple barriers still exist prior to a broad introduction of NOTES into clinical practice. However, if the concept of transluminal surgery is supported by the use of one or two tiny transcutaneous instruments, transluminal surgery could be significantly facilitated and enhanced. This type of combined or "hybrid" surgery (denominated as well as "rendezvous" technique) is not entirely new. The first reports have already been published a few years ago [10]. Originally, the endoscopist mainly supported the laparoscopist in performing the surgery (Fig. 3).

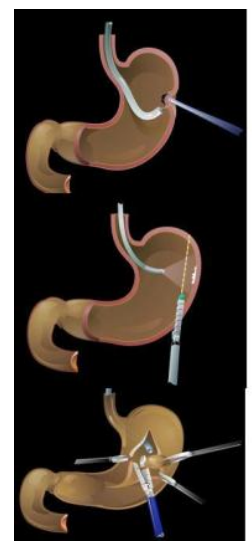

Fig. 3: Local excisions at the stomach (left) and colon (right) using combined endoluminal/intracavitary procedures.

Today, the endoscopist could take over the dominant role and the transcutaneous role could be confined to exposure or other auxiliary tasks. Thus, even frequently performed operations such as cholecystectomy could be performed without almost any major scar There is no doubt that laparoscopically assisted NOTES is ready for clinical use and many of the tasks of CS as mentioned above could already be realized in hybrid surgery.

The problem of spatial orientation is even more important here than in pure NOTES operations, since the laparoscopist (the surgeon who handles the percutaneous instruments) is confronted with the problems that develop when working off the axis of the camera angle. Many hybrid procedures are performed with the endoscope in a retroflective position and the image "upside down". With experience, some of this spatial incongruity can be overcome as it could be learned with the introduction of combined operations, but it will prevent complex procedures from being performed with the same speed and facility that total in-line visualization would allow. Automatic electronic image stabilization is a first step (Fig.
4), but translating the intended actions into a virtual straight-forward view when the telescope and the actuators are in a retroflected position is the next issue for CS.
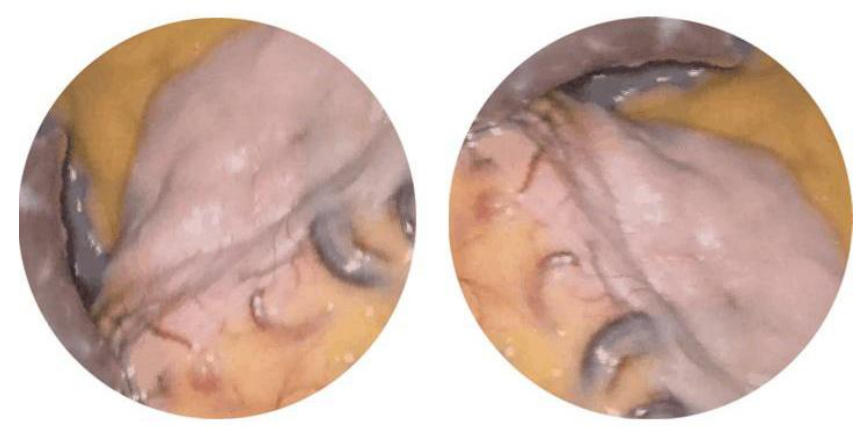

Fig. 4: Endoscopic view of the stomach: Left: Original image as produced by the endoscope Right: The same image is rotated clockwise into the position the laparoscopist is accustomed to.

The use of augmented reality, based upon preoperative diagnostic work up and intraoperative navigation, would make surgeries safer and more efficient, since normal anatomy and pathologies could be made visible both for the "external" and the "internal" partner. Intraoperative navigation, in general, could optimize the required close cooperation (Fig. 5).

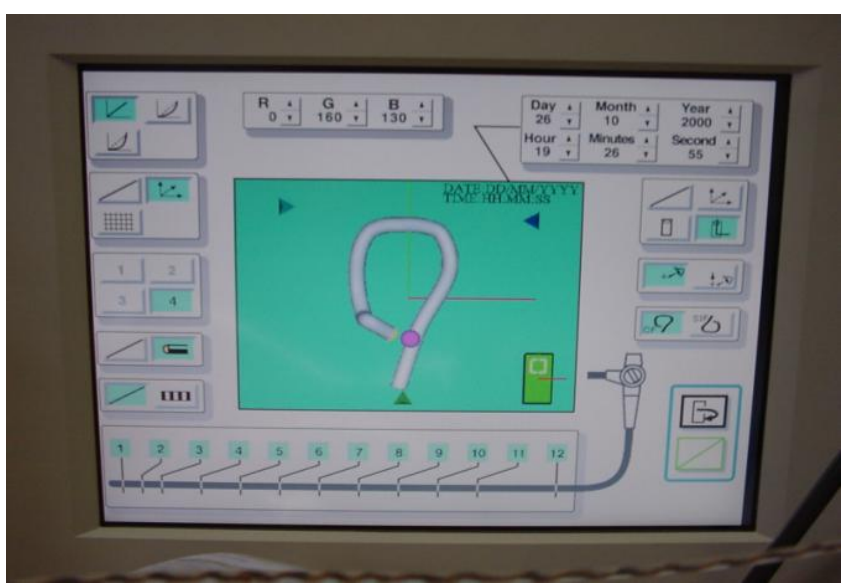

Fig. 5: The shape and the position of a colonoscope in situ are made visible by multiple electromagnetic sensors integrated into the flexible instrument [11].

Many of the essential advances which are expected from CS to make NOTES feasible for clinical routine could already be achieved on the basis of hybrid procedures which are already performed now. General OR management is just one topic (Fig. 6). NOTES will require even more than hybrid procedures the integration of a large number of 
various devices and systems with a high degree of standardization and a uniform user-system interface.

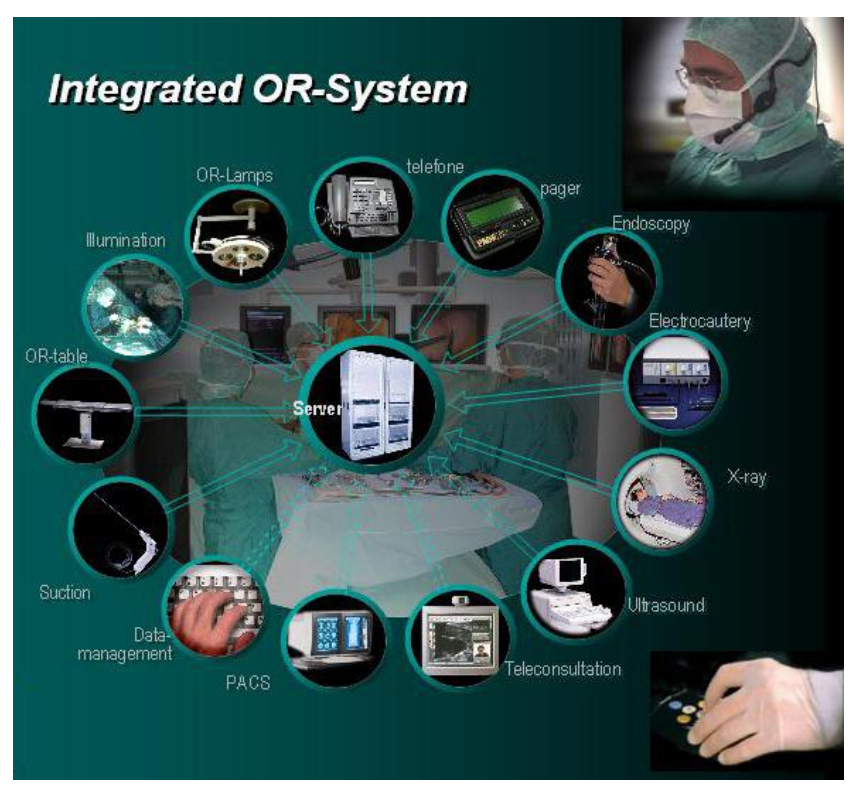

Fig. 6: Comprehensive control of all relevant OR systems by the surgical team [4].

Though differing from NOTES in some technical regards, hybrid procedures already allow one to establish continuous automated data collections, since many sources are identical. Some important information is even available today (Fig. 7).

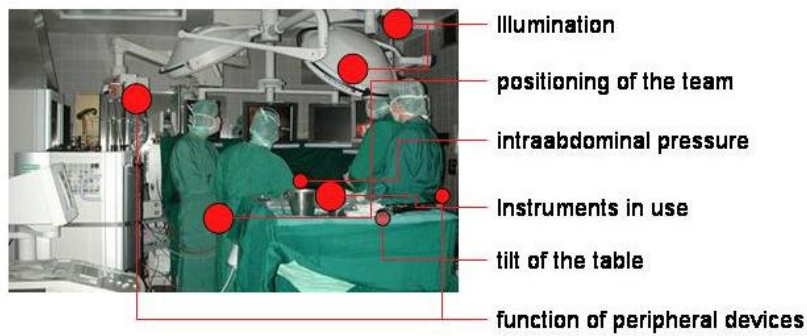

Fig. 7: Online data retrieval during hybrid surgery (MITI)

Last but not least, automated documentation could be gradually introduced into the daily routine, not only to render documentation more comprehensive and precise but also to reduce the work load of the staff. In addition, clinical evaluation of these surgical techniques according to the requirements of evidence based medicine could be significantly enhanced by new tools of information storage, retrieval, and analysis [12].

\section{REFERENCES}

[1] D. Rattner, A. Kalloo, and the SAGES/ASGE Working Group on Natural Orifice Translumenal Endoscopic Surgery, "ASGE/SAGES Working Group on Natural Orifice Translumenal Endoscopic Surgery”, Surg Endosc, 20:329-333, 2006

[2] K.G. Vosburgh, and R.S.J. Estépar, "Natural Orifice Transluminal Endoscopic Surgery (NOTES): An opportunity for Augmented Reality", Stud Health Technol Inform, 125:485-490, 2007

[3] L.L. Swanstrom, R. Kozarek, P.J. Pasricha, S. Gross, D. Birkett, P.O. Park, V. Saadat, R. Ewers, and P. Swain, "Development of a new access device for transgastric surgery", J Gastrointest Surg, 9(8):1129-1136, 2005

[4] H. Feussner, "The operating room of the future: A view from Europe", Semin Laparosc Surg, 10(3):149-156, 2003

[5] J.V. Sutherland, W.-J. van den Heuvel, T. Ganous, M.M. Burton, and A. Kumar, "Towards and intelligent hospital environment: OR of the future", Stud Health Technol Inform, 118:278-312, 2005

[6] S. von Delius, W. Huber, H. Feussner, D. Wilhelm, A. Karagianni, J. Henke, A. Preissel, A. Schneider, R.M. Schmid, A. Meining, "Effect of pneumoperitoneum on hemodynamics and inspiratory pressures during natural orifice transluminal endoscopic surgery (NOTES): An experimental, controlled study in an acute procine model", Endoscopy, 39:854-859, 2007

[7] N. Padoy, M. Horn, H. Feussner, M.O. Berger, and N. Navab, "Recovery of surgical workflow: a model-based approach", Int J CARS, 2(Suppl 1):S479-S482

[8] S. Gillen, E. Doundoulakis, A. Schneider, A. Fiolka, S. von Delius, A. Meining, and H. Feussner, "The ELITE manikin: Construct validation of a new training system for Natural Orifice Translumenal Endoscopic Surgery (NOTES)", Gastrointest Endosc, (submitted), 2007

[9] E. Johnson, "Surgical simulators and simulated surgeons: Reconstituting medical practice and practitioners in simulations", Soc Stud Sci, 37(4):585-608, 2007

[10] H. Feussner, D. Wilhelm, V. Dotzel, D. Papagoras, and E. Frimberger, "Combined endoluminal and endocavitary approaches to colonic lesions", Surg Technol Int, XI:97-101, 2003

[11] S. von Delius, H. Feussner, R.M. Schmid, E. Frimberger, "Is electromagnetic navigation useful for routine colonoscopy?", Endo heute, 19:23-25, 2006

[12] Boye , N. Eberholst F. Farlie, R. Sorensen, LB. Lyng $\mathrm{KM}$, „User driven evidence based experimental design: anew method for interface design used to develop an interface for clinical overview of patient records", Medinfo $121053-1057,2007$ 